\title{
INTEGRATING MOBILE ROBOTS DEVELOPMENT AND COMPETITIONS IN ENGINEERING CURRICULA
}

\author{
C. Cardeira, J. M. C. Sousa, J. R. Caldas Pinto, M. Ayala Botto, M. \\ Ramalho, J. Sá da Costa \\ Technical University of Lisbon,Instituto Superior Técnico, GCAR-IDMEC \\ Av. Rovisco Pais, 1049-001 Lisbon, Portugal \\ \{cardeira@dem.,jmsousa@,jcpinto@dem.,ayalabotto@,mar@dem., \\ sadacosta@dem.\} ist.utl.pt
}

\begin{abstract}
In this paper we present an experience to integrate mobile robots competitions in engineering courses at the university level. We present the challenges provided by mobile robot competitions, the mobile platform we developed, the courses involved and the research contributions of this work. This experience has been conducted at Technical University of Lisbon, Portugal, and is now on its second year of implementation. Copyright (C) IFAC 2006.
\end{abstract}

Keywords: Mobile Robots, Robotic Competition, Education

\section{INTRODUCTION}

The use of robots in education is becoming a common practice in engineering courses. Over 100 competitions involving robots currently exist (Osuka 2002), providing entertainment value, improving science education in schools and science dissemination to the general public.

One of the most critical challenges when organizing such a competition is the balance between science and entertainment. Media attention is very sensible to the entertainment value and media attracts sponsors. Some competitions are purely media oriented like some remote operated robot contests regularly held on televisions. The disadvantage of pure entertaining competitions is the marginalization of the technical contribution (Osuka 2002).

\subsection{Robotic competitions for education and research}

Other competitions are more science oriented, involving much more research relevance (Bräunl 1999).
Robocup (Kitano 1998), (Marques 2004) requires autonomous robots without neglecting the entertainment value by the association of robots to soccer.

Many science associations or governmental agencies promote annual editions of robotic contests like the AAAI Mobile Robot Competition (Michaud 2001), (Elinas 2002), the Association of Unmanned Vehicle Systems, (Braunl 1998), (Manseur 2000), or DARPA which promotes the Darpa Grand Challenge (DGC) giving a 2 million dollar award to the winner (if any) of a 175 miles desert road race from Los Angeles to Las Vegas including dirty roads, trails, open desert and man-made obstacles (Murray 2005). The price for the 2005 edition was awarded for the first time as five autonomous robots finished the race. Many universities run their own local competitions as part of their educational activities (Rieber 2004), (Almeida 2000). Some universities use standard available entertainment platforms like Lego Mindstorms and their RCX controllers. For instance, the Department of Mechanical Engineering at the University of Stuttgart uses LEGO robots to illustrate feedback control problems with competitions based in path following or active suspension control problems (Rieber 2004). 
With or without competitions, the truth is that the use of robots on education and research (mobile or not) is increasing and many institutions already use them for education and research purposes (Schilling 2002), (Bruder 2003), (Lima 1998), (Weinberg 2003), (Ceccarelli 2003).

\subsection{Our goal}

It is our goal to find a good balance between the entertainment value and the technical contribution of the work. To achieve this goal we had to define a more or less standard platform that the competitors should use, according to the university curriculum and to our team research goals.

The Mobile Robot Platform section presents the standard platform we started from. The Curriculum Integration section presents how several courses used this platform. The Competition and Students Feedback section presents how we ran the competition and the feedback we got. Section Research relevance presents our goals of integrating the competition of the research areas of our team and finally, in the last section, some conclusions are drawn.

\section{MOBILE ROBOT PLATFORM}

\subsection{Common practices}

Lego Mindstorms are a common solution for building mobile robots. They undoubtedly present a good entertainment value and provide an easy-to-use and flexible enviroment (Rieber 2004), (Weinberg 2003). It is true that the programming environment available for these robots is improving everyday. Looking inside Lego Mindstorms we may see that the RCX control module is based on a 8 bit 16KB ROM, 28KB RAM Hitachi H8/3292 microcontroller with 3 analogue inputs and 3 analogue outputs (Gawthrop 2004), (Polpeta 2003). It is programmable in several languages including $\mathrm{C}$.

However we feel that, in the actual state, the use of the RCX controllers has some limitations for our purposes. Despite the number of $\mathrm{I} / \mathrm{O}$ ports is limited, it is still enough for many applications. But, in today's universities and industry it is very common that students and engineers are acquainted with several programming languages (.NET, Java) or some engineering programming environments like Matlab, Simulink or Labview. A platform that could use these languages and environments would provide a more straightforward connection from theory to practice.

Small microcontrollers like Microchip or Atmega families provide a very interesting alternative. They have plenty of I/O possible configurations, there are a lot of programming environments for them, and they are very low cost. They have by far less entertainment value than a Lego RCX controller but the technical contributions we might achieve with the use of such microcontrollers are undoubtedly higher. But again the programming capabilities are limited to the existing cross compilers available. Moreover they provide good solutions for analogue or digital I/O ports but not for directly handling cameras for image processing.

\subsection{Our Approach}

To achieve a flexible platform we decided to use a normal laptop as the processing unit and a low cost USB card for I/O interconnections. We made this approach because laptops are more and more common among university students. For instance, the OLPC ("One Laptop per Child”) - project aims to deliver laptop computers for less than 100 dollars each unit (Negroponte 2005). Therefore, it is very common that among two or three students in a group, at least one has a laptop. So the students use their own laptops to control the robots. Students complain less about the equipment when they use their own laptops, which in many cases are updated and powerful. They are responsible for damages so they pay a lot more attention to the programs they run and the hardware they connect to. Moreover, the robot uses a normal webcam for guidance which the students usually already have.

Students may use the languages and operating systems available for laptops. The use of Visual Studio, Matlab/Simulink, Labview, Linux based solutions, etc, become easily possible. Comparing to other common practices, we may say that tasks like image processing, visual servoing or system identification can be done inside a normal laptop but are not straightforward or sometimes impossible with actual Lego Mindstorms. When compared to microcontrollers, it is true that microcontrollers provide good solutions for analog or digital I/O ports but not for handling image processing cameras. It is actually possible to do that (Kaiser 2001), but it is not as straightforward as connecting a webcam to a USB laptop port.

\subsection{Hardware}

Taking into account the above pre-conditions we built a low cost mobile robot with commercial off-the-shelf components. The cost depends on the choices of some components but the overall cost of the robot is under 200 Euros (of course, this price does not include the laptop cost, which we consider already available).

The first robot of the series, so called "Rasteirinho", is described in more detail in (Cardeira 2005). It uses a low cost USB card and motors, batteries and chargers of disassembled low cost electrical screwdrivers and drills. The laptop is easily attached to or removed from the robot because it is mechanically fixed to the robot by easily detachable Velcro bands. Moreover, the only electrical connection to the robot is through a single cable to the USB port. 
Figure 1 shows the mobile robot built where we may see several robot components and the final assembly. The advantage of such a simple robot is that it becomes possible to lend the robot to the students freeing the laboratories occupancy and providing a new challenging and unusual peripheral for the students own laptops.
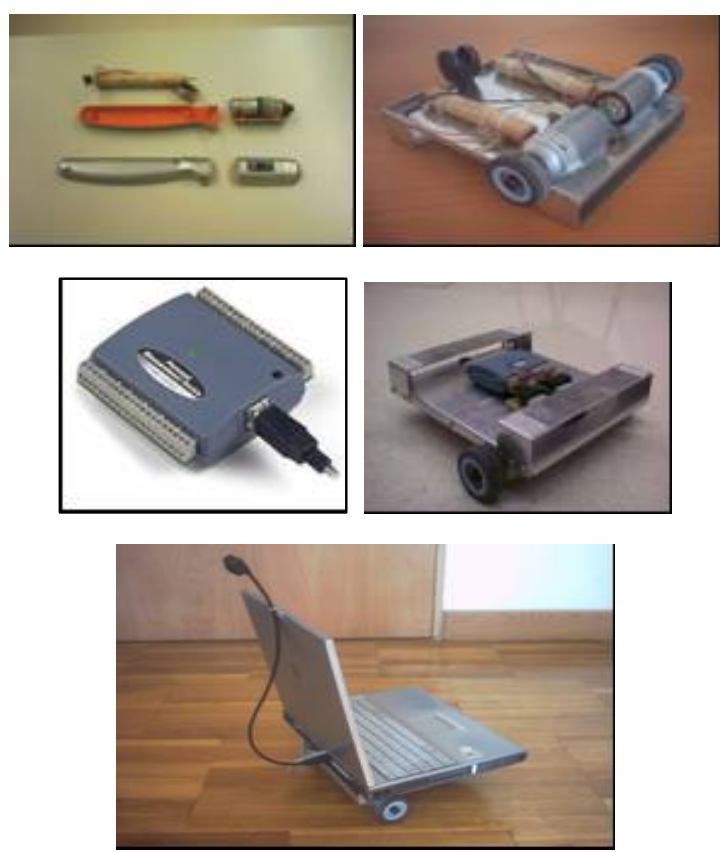

Figure 1: Mobile Robot built

\subsection{Software}

We developed a baseline package of software that is able to run the mobile robot and make it perform several tasks like a line or target follower. The standard package provides a common background for all the students and it includes the following components: test programs (with source) to check if the actuators actually respond correctly; test programs (with source) to check if the image acquisition is performing correctly; a program (with source in Visual Studio .NET using C\#) with the real-time computation of an image mass centre; a program, with source, using the above modules to perform a line follower.

This information is freely available for students (IARC 2005) and, upon compilation of the above sources, the students have a standard platform that works. Each course will then focus on a specific subject in which the students produce their own work. For the subjects not connected to the course the student may use the standard solutions available on the baseline package.

Figure 2 presents the robot with the standard package installed and performing a line follower task.
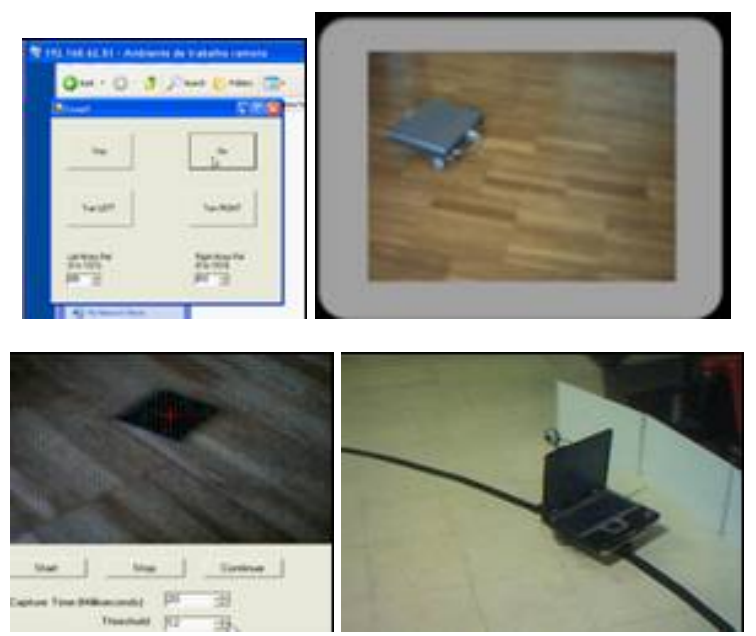

Figure 2: Test software and a line follower

\section{CURRICULUM INTEGRATION}

Several universities already extended this practical view to their curriculum contents (Nagai 2001). In IST the experience is being conducted in several courses of both Mechanical Engineering and Computer Science Engineering graduations. We encourage the formation of mixed teams as long as the supervisors of the courses agree upon the contribution of each student. Several courses used this robot (the number of available robots is now about 10 but a number of 100 available robots is sough) for partial evaluation of their students. In the following subsections we present some of the achieved results in the following courses: Systems and Signals, Control Systems, Industrial Computing, Introduction to Programming, Advanced Programming and Artificial Vision.

\subsection{Systems and Signals}

The Systems and Signals course is in core of the Computer Science Engineering curriculum (third year). The course focuses on examples and definitions of Signals and Systems, state machines, composing state machines, linear systems, frequency domain, frequency response, filtering, Fourier transforms, sampling and reconstruction.

The course follows the Berkeley approach (Lee 2000a,b) (Lee 2003) (Costa 2005) and has a relevant laboratory. Some students were chosen for doing their laboratory in these mobile robots platforms. The main goal was to find the structure of signals and systems presented. Students defined the functions for signals and the systems as functions of functions. For instance, an image is a function: Image: VerticalSpace $x$ HorizontalSpace $\rightarrow$ Intensity;

A pattern recognition system that processes an image to find the coordinates $x, y$ of the position and the $\alpha$ orientation of a given pattern presented in the image, is defined as:PatternRecognition: Image $\rightarrow\{x, y, \alpha\}$ 
These simple concepts were illustrated with the mobile robot platform. For the competition, Systems and Signals students were able to use more straightforward tools than the hard C\#. Students were divided by the use of Matlab or Labview. In the competition, the robot had to accomplish the mission of autonomously going from one laboratory to the other by using the minimum number of arrows on the floor (see Figure 3).

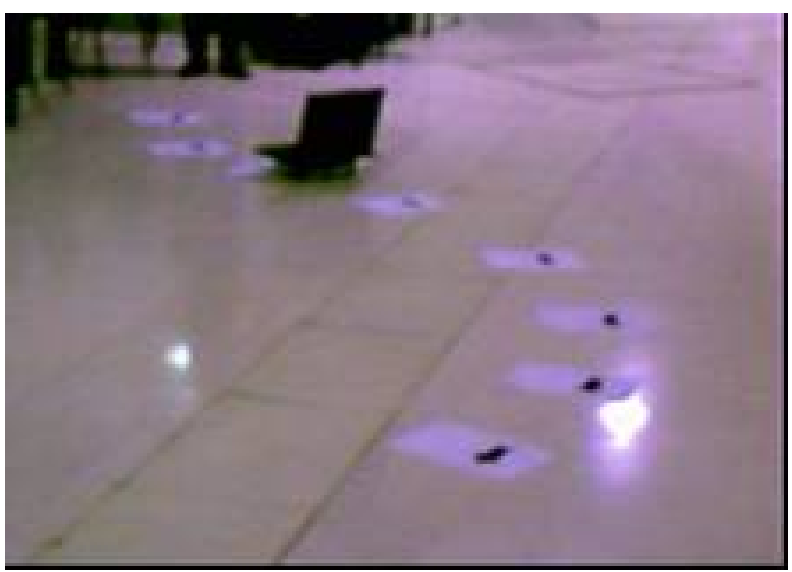

Figure 3: The robot guided by the arrows

The image toolbox of Labview (IMAQ Vision) performed very nicely, detecting the arrow patterns with normal webcams (see Figure 4). The $\{x, y, \alpha\}$ information was used both to detect the path the robot had to follow and to have a practical example of the PatternRecognition system definition.

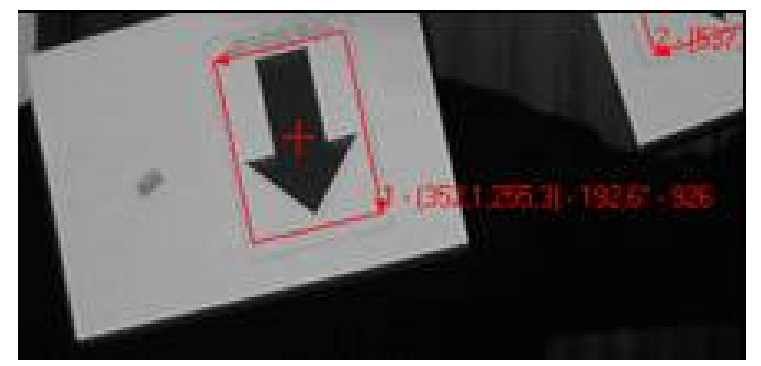

Figure 4: Testing pattern recognition

\subsection{Control Systems}

The Control Systems course belongs to the 4th year of the Mechanical Engineering curriculum. It focuses on the analysis, design and tuning of feedback controllers (PID, lead-lags, master-slave), feedforward controllers, as well as on the control of systems with time-delays (the Smith predictor). It also includes the design of digital control systems.
In this course the students designed and tuned two digital PID controllers and implemented them in the robot in the line follower problem. These controllers, working independently, were able to control the torque applied to each wheel while stabilizing the distance to the target. A simple software program was also developed for the online helping of the designer in setting up the best controllers' parameterization. At the end, students agreed to consider this experience very fruitful, since they could actually be involved in a real control system design experiment.

\subsection{Industrial Computing}

The Industrial Computing course is optional for the last year students of Mechanical Engineering. In this course, students interact computers to with physical world through several platforms. A group of students participated in the competition with a pure Matlab/Simulink implementation. The goal was not to score better but to easily benefit from Simulink declaration language providing much more clarity than the Matlab imperative language.

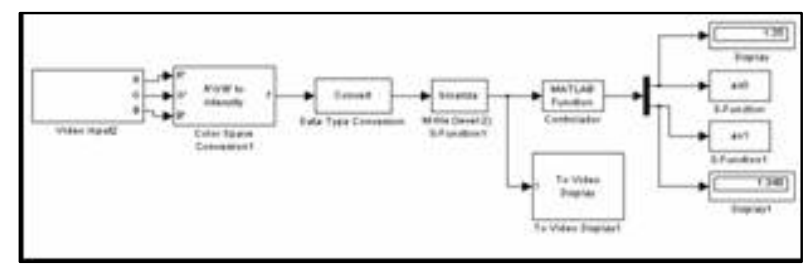

Figure 5: Simulink blocks to control the line follower

In this work students used Simulink blocks to control the line follower and it became much more intuitive than hundreds of lines of code. Moreover, changing the controller to a different one is easy as it demands just the change of the controller block allowing the use of different controllers with different performances.

\subsection{Introduction to Programming}

The Introduction to Programming course is offered to the Mechanical Engineering first year students. The main goal of this course is to supply the ability of developing wellstructured algorithms and programs in a computational language. The course dedicated some weeks to teach Matlab. As a pilot experience, four groups of two students implemented an algorithm to follow a line. Some modules were distributed to the students, namely, the module that detects an image from a webcam and compute its center of gravity, and a basic controller in the Simulink environment. The students had to join the modules and program the remaining parts of the algorithm. When coding their controller, these students felt that according to the parameters the robot may perform better or worse (some times the 'worse' was actually unstable). Further, they interacted with older students, which had robots performing naturally much better and with better stability. This 
fact increased largely the first year students interest for the courses that they will have to follow later, showing in practice their importance in 'real world' applications.

\subsection{Advanced Programming}

The Advanced Programming course belongs to the curriculum of the Mechanical Engineering ( $4^{\text {th }}$ year). The course focus object oriented modeling and programming centered in on the $\mathrm{C}++$ language. How Matlab deals with objects is also studied. We use image processing algorithms due to its increasing importance in engineering in general and, in particular, in mechanical engineering. Both teaching goals find an excellent final application on the mobile robots competitions. Students are encouraged to develop their own image processing algorithms and improve the overall platform. This work allows also a smooth introduction in Windows based programming, which does not belong to the core of the course as it occurs in the majority of the programming courses. Finally, it is always very compensating for students to see that they manage to put "things" working in practice with the tools that they have just learned.

\subsection{Artificial Vision}

The Artificial Vision Course is optional for 5th year students. It is aimed for applications towards Machine Intelligence, e.g., Robot vision, Automatic Inspection and Autonomous Machines. Among these, autonomous vehicles are a good basis for work. Different tasks can be performed in different environment conditions which can be controlled to a certain degree. Applications to drive the platform in different environment conditions were implemented, mainly to follow to a target avoiding sparsely obstacle randomly positioned. Examples are presented in Figure 6, where driving through a luminous target is made in a corridor with shinning floor and mixed illumination conditions.

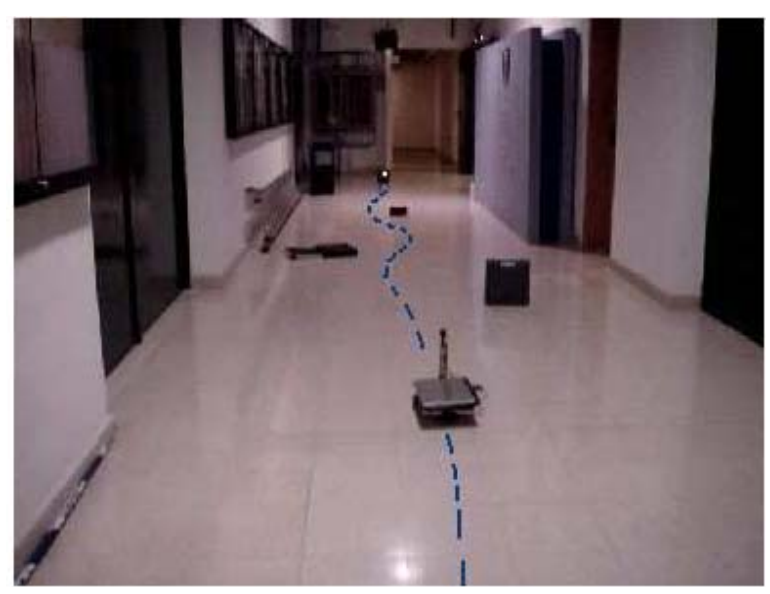

Figure 6: Obstacle avoidance

\section{COMPETITIONS AND STUDENTS FEEDBACK}

A local robotics competition (IARC) is organized at the end of each semester. In June 2005 we had the third edition. In the first edition, the contest consisted of two parts: a free demonstration and the actual competition. The robot had to follow a target that consisted of a black CDROM box placed over the floor and attached to a preprogrammed mobile robot. By using a preprogrammed robot to pull the CD box we ensured a fair competition in the sense that all the teams performed in the same conditions. In the second edition there were no more targets to follow. The robot had just to make a trajectory based only on arrows placed in the floor indicating the direction to follow. In the third edition, first year students participated for the first time and we came back to a line follower but with the possibility of departure from a "parking place" outside the track to follow (Cardeira 2005).

This educational experience is not yet implemented for a large number of students. The number of available robots is growing up but it is still limited and we are not yet in position to freely lend them to the students. Hence the feedback is not yet representative but the students' reaction is mainly positive. What they actually learn depends on the course they are following and the different experiences of the team members.

Students of introductory courses learn that their lines of code can make much more than reading keyboards and sending graphics to the screen; they actually can make a robot move.

In more advanced courses, like control courses, learn in practice how to tune their controllers to keep the robot following the target steadily and smoothly. In any case, the participation on these contests provides a very practical experience on the programming languages they use like C\#, Matlab/Simulink or Labview.

\section{RESEARCH RELEVANCE}

The objective for competitions must remain within the research interests of a particular area. The entertainment value is important but it is very disappointing to put an enormous research effort reduced to performance in a particular event.

A competition has to be a part of a larger and well organized research program. It is also important to participate in competitions that are embedded on larger conferences, because it allows the presentation of the research behind the competition, in the right forum to discuss related ideas (Bräunl 1999).

These mobile platforms can perform as a testbed for interesting research problems including cooperative work, multi-robot and multi-agent scenarios, sensor fusion, planning, real-time recognition and reasoning, real-time 
image tracking (Cardeira 2005), localization (Alves 1998) and navigation (Borges 1998).

\section{CONCLUSIONS}

We presented low cost robots and their usage for the integration in the curricula of engineering courses. We felt that they create student interest and inspire them to dedicatedly work on other projects. The practical solutions students find for all the small problems encountered in preparing a robot for a competition will teach skills that a normal lecture ever could (Bräunl 1999). These platforms seem to be a good complement for uni-versity laboratories. Letting the student bring home the robots will increase the time they stay in contact with the experiments, while giving a totally unexpected use to their personal laptops (Cardeira 2005).

\section{REFERENCES}

Almeida (2000) Almeida, L., Azevedo, J., Cardeira, C., Costa, P., Fonseca, P., Lima P., Ribeiro, F., Santos, V., "Mobile Robot Competitions: Fostering Advances in Research, Development and Education in Robotics", in Proceedings of CONTROLO'2000, the 4th Portuguese Conference on Automatic Control, Guimarães - Portugal, October 4-6, 2000, pp. 592-597

Alves (1998) Alves, R.; Borges, J., Lima, R., Pasadas, F., Cardeira, C.; "An Algorithm for Total Filling fo an Unknown Surface: Analysis and Simulation" in Proceedings of EUREL'98 European Advanced Robotics System Development: Mobile Robotics, Leiria, Portugal, September 14-16, 1998.

Braunl (1999) Braunl, T., Research relevance of mobile robot competitions, Robotics \& Automation Magazine, IEEE, Volume 6, Issue 4, Dec. 1999, pp. 32 - 37

Borges (1998) Borges, J., Lima, R., Alves, R.; Pasadas, F., Cardeira, C.; "Triangulation Method for Determination of a Robot's Location" in Proceedings of EUREL'98 European Advanced Robotics System Development: Mobile Robotics, Leiria, Portugal, September 14-16, 1998

Bruder (2003) Stephen Bruder and Kevin Wedeward, Robotics in the Classroom, IEEE Robotics \& Automation Magazine, Volume 10, Issue 3, September, 2003, pp. 25-29

Cardeira (2005) Carlos Cardeira, José Sá da Costa, A Low Cost Mobile Robot for Engineering Education, in Proceedings of IECON 2005, the 31st Annual Conference of the IEEE Industrial Electronics Society, Raleigh, USA, November 2005, pp. 2162 - 2167

Ceccarelli (2003) Ceccarelli, M., Robotic Teacher's Assistants: Low- Cost Robots for Research and Teaching Activities, IEEE Robotics \& Automation Magazine, Volume 10, Issue 3, Sept. 2003, pp. $37-45$

Costa (2005) Sérgio Costa, Carlos Cardeira, João Pargana, F. Miguel Dionísio, Pedro A. Santos, "Systems and Signals Online Questions and Grading”, in Proceedings of ETFA 2005, the 10th International Conference on Emerging Technologies and Factory Automation, Catania, Italy, September 2005, pp 41-47

Elinas (2002) Elinas, P.; Hoey, J.; Lahey, D.; Montgomery, J.D.; Murray, D.; Se, S.; Little, J.J., "Waiting with Jose, a visionbased mobile robot", in Proceedings of ICRA '02 the IEEE
International Conference on Robotics and Automation, Volume 4, May 2002, pp. 3698 - 3705

Gawthrop (2004) Gawthrop, P.J.; McGookin, E., A LEGO-based control experiment, IEEE Control Systems Magazine, Volume 24, Issue 5, Oct. 2004, pp. 43 - 56

IARC (2005) http://www.dem.ist.utl.pt/ carclub/Clube/ (in portuguese)

Kaiser (2001) J. Kaiser, P. Schaeffer: "ICU - A smart optical sensor for direct robot control", in Proceedings of the IEEE International Conference on Mechatronics and Machine Vision in Practice, Hong Kong, China, August 27-29, 2001

Kitano (1998) Kitano, H.; Asada, M.; Noda, I.; Matsubara, H., RoboCup: robot world cup, IEEE Robotics \& Automation Magazine, Volume 5, Issue 3, Sept. 1998, pp 30 - 36

Lee (2000a) Edward A. Lee and Pravin Varaiya, "Introducing Signals and Systems -- The Berkeley Approach," in Proc. of the First Signal Processing Education Workshop, Hunt, Texas, October 15 - 18, 2000.

Lee (2000b) Edward A. Lee, " Designing a Relevant Lab for Introductory Signals and Systems," in Proc. of the First Signal Processing Education Workshop, Hunt, Texas, October $15-18,2000$

Lee (2003) Edward A. Lee and Pravin Varaiya, Structure and Interpretation of Signals and Systems, Addison-Wesley, 2003.

Lima (1998) Lima, P.; Cardeira, C.; The MiniRobot Project: Learning from Building Small Mobile Robots, IST Science \& Tecnology, N. 3, December 1998, pp. 16-22.

Manseur (2000) Manseur, R., "Hardware Competitions in Engeneering Education", in Proceedings of the $30^{\text {th }}$ ASEE/IEEE Frontiers in Education Conference, Volume 2, October 2000 pp. F3C/5 - F3C/8

Michaud (2001) Michaud, F.; Audet, J.; Letourneau, D.; Lussier, L.; Theberge-Turmel, C.; Caron, S., Experiences with an autonomous robot attending AAAI, IEEE Intelligent Systems, Volume 16, Issue 5, Sep-Oct 2001, pp 23 - 29

Marques (2004) Marques, C.; Lima, P., Avoiding obstacles multisensor navigation for nonholonomic robots in cluttered environments, Robotics \& Automation Magazine, IEEE, Volume 11, Issue 3, Sept. 2004, pp $70-82$

Murray (2005) Murray, R.M., "Autonomous Machines: Racing to Win the DARPA Grand Challenge", in Proceedings of the American Control Conference, June 8-10, 2005, pp. 9-10

Nagai (2001) Nagai, K., Learning while doing: pratical robotics education, IEEE Robotics and Automation Magazine, Volume 8, Issue 2, June 2001, pp. 39-43

Negroponte (2005) Negroponte, N.. “\$100 laptop”, MIT Media lab, http://laptop.media.mit.edu/, 2005.

Osuka (2002) Osuka, K.; Murphy, R.; Schultz, A.C.; USAR competitions for physically situated robots, IEEE Robotics \& Automation Magazine, Volume 9, Issue 3, Sept. 2002, pp. 26-33

Polpeta (2003) Polpeta, F. ; Fröhlich, A., LEGO RCX - Hitachi H8/3292, LISHA/UFSC, http://www.lisha.ufsc.br/ guto/ teaching/ish/processors/h8/overview.pdf, Mars 2003.

Rieber (2004) Rieber, J.M.; Wehlan, H.; Allgower, F., The ROBORACE contest: Using LEGO robots to teach the fundamentals of feedback control, IEEE Control Systems Magazine, Volume 24, Issue 5, Oct. 2004, pp. 57 - 60

Schilling (2002) Schilling, K; Roth, H; Rösch, O, Mobile MiniRobots for Engineering Education, Global J. of Eng. Educ., Vol 6, No. 1, pp 79-84.

Weinberg (2003) Weinberg, J.; Yu, X., Robotics in Education: Low Cost Platforms for Teaching Integrated Systems, IEEE Robotics \& Automation Magazine, Volume 10, Issue 3, Sept. 2003, pp. 3-6. 